\title{
Complementary Effect of Blending on the Volatile Composition of Albariño and Loureira White Wines (Vitis vinifera L.) from NW Spain
}

\author{
M. Vilanova ${ }^{1 *}$, L. Freire ${ }^{2}$ \\ (1) Misión Biológica de Galicia (CSIC), El Palacio-Salcedo, 36143, Pontevedra, Spain \\ (2) Santiago Ruiz Winery, 36760 O Rosal, Pontevedra, Spain
}

Submitted for publication: October 2016

Accepted for publication: January 2017

Key words: Blended wine, volatiles aroma; Albariño, Loureira, Caiño blanco, Godello

\begin{abstract}
Albariño and Loureira are highly valuable white grape cultivars from the Northwest Iberian Peninsula (Northwest Spain and Northern Portugal). The purpose of this study was to determine how blending affects the volatile composition of Albariño and Loureira white wines. Four Vitis vinifera white wines (Albariño, Loureira, Caiño Blanco and Godello) were blended at different proportions, resulting in four wines two two-wine blends (83:17 Albariño-Caiño Blanco and 50:50 Loureira-Caiño Blanco) and two threewine blends (48:32:20 Albariño-Loureira-Caiño Blanco and 35:35:30 Loureira-Caiño Blanco-Godello). The blended wines were compared with Albariño and Loureira single wines. The volatile composition of the wines was evaluated using gas chromatography-mass spectrometry to identify changes in the aroma compounds of the blends with respect to the Albariño and Loureira varietal wines. The Loureira and Albariño single wines had the highest concentrations of volatiles. However, the blended wines, especially those with Loureira, showed increases in terpenes and $\mathrm{C}_{13}$-norisoprenoids. Discriminant analysis showed that $\mathrm{C}_{6}$-alcohols and phenol volatiles were the variables that contributed the most to the differences between Albariño and the blended wines, whereas terpenes and $C_{13}$-norisoprenoids made the greatest contributions to the differences between Loureira and the blended wines. The odour activity value was calculated to analyse the sensory influence of the volatiles, and this showed the superiority of the Loureira single wine aroma and the 50:50 Loureira-Caiño Blanco blended wines vs. Albariño and the other blended wines. The blended wines with a high proportion of Loureira appeared to be richer in volatile compounds, which increased the complexity of the wines' aroma.
\end{abstract}

\section{INTRODUCTION}

Blending wines is a common practice in red wine production; however, white wines have always been valued more as monovarietals. In recent years there has been a tendency to seek new products from the blending of white wines to achieve greater complexity. Aroma compounds play an important role in the quality and complexity of wine, because these compounds produce an effect on sensory perception (Francis \& Newton, 2005). The most important wine volatile compounds include those from different families, such as alcohols, $\mathrm{C}_{6}$-compounds, terpenes, $\mathrm{C}_{13}{ }^{-}$ norisoprenoids, volatile fatty acids, ethyl esters, acetates, phenol volatiles and other compounds. The concentration of volatile compounds in a wine depends of the grape cultivar, the terroir conditions and winemaking techniques (Vilanova et al., 2007; Herderich et al., 2015). Blending of wines is an ancient technique used in wine-growing regions. Wines are blended for several reasons, including increasing complexity, maintaining a consistent character and quality, and developing winery-typical wines (Hopfer et al., 2012).

The Northwest Iberian Peninsula specialises in the production of white wines. Galicia (Northwest Spain) and North Portugal have established monovarietal and blended wines from different aromatic white cultivars, such as Albariño, Loureira, Godello and Caiño Blanco, with Albariño being the most widely grown cultivar. Recently, Albariño has become an important cultivar in other countries in Europe, the United States and Australia.

There have been several studies on the volatile composition and sensory characteristics of white grape cultivars, musts and wines from different geographical areas in Galicia and Portugal. These works include those on Albariño (Versini et al., 1994; Carballeira et al., 2001; Vilanova \& Masneuf-Pomarède, 2005; Vilanova \& Vilariño, 2006; Vilanova et al., 2007; 2008; 2010), Loureira (Versini et

*Corresponding author: E-mail address: mvilanova@mbg.csic.es

Acknowledgements: We would like to thank Dr Manuel Marcos from CACTI (Vigo University) 
al., 1994; Oliveira et al., 2004; 2008), and Godello (Versini et al., 1994; Vilanova, 2006; Losada et al., 2011). However, how blending affects the volatile composition of these white wines has not yet been studied.

In this study, compounds responsible for the varietal and fermentative aromas (terpenes, $\mathrm{C}_{13}$-norisoprenoids, alcohols, $\mathrm{C}_{6}$-alcohols, ethyl esters, acetates, volatile fatty acids, phenol volatiles and other compounds) of Albariño and Loureira single wines and blends with Caiño Blanco and Godello white wines were studied. The aim of this work was to determine if different two-wine blends (AlbariñoCaiño Blanco, Loureira-Caiño Blanco) and three-wine blends (Albariño-Loureira-Caiño Blanco, Albariño-Caiño Blanco-Godello), as complementary wines for Albariño and Loureira, produce significant changes in the wines' aroma. In this study, individual volatile compounds and odour activity values $(\mathrm{OAVs})$ were determined in the wines.

\section{MATERIALS AND METHODS}

\section{Wine samples and blending}

Four Vitis vinifera white grape cultivars (Albariño, Loureira, Caiño Blanco and Godello), grown in a vineyard from the Galicia region (Northwest Spain), were harvested at their optimal ripeness $\left(22\right.$ to $\left.23^{\circ} \mathrm{Brix}\right)$. The monovarietal wines were produced in the Santiago Ruiz winery (AOC Rías Baixas, Northwest Spain), as follows: after pressing, the musts were placed into $15000 \mathrm{~L}$ stainless steel tanks. Before fermentation, sulphur dioxide $(120 \mathrm{mg} / \mathrm{L})$ was added to the musts. All fermentations were conducted using the same Saccharomyces cerevisiae commercial yeast at $18^{\circ} \mathrm{C}$. After fermentation, the wines were blended at different proportions, resulting in six wines (Table 1):
- Two single wines: 100\% Albariño (AL) and 100\% Loureira (LO),

- Two two-wine blends: 83\% Albariño and 17\% Caiño Blanco (83:17 AL-CB), and 50\% Loureira and 50\% Caiño Blanco (50:50 LO-CB), and

- $\quad$ Two three-wine blends: 48\% Albariño, 32\% Loureira and 20\% Caiño Blanco (48:32:20 AL-LO-CB), and $35 \%$ Loureira, 35\% Caiño Blanco and 30\% Godello (35:35:30 LO-CB-GO).

One month after blending, three samples of each wine were transferred to $500 \mathrm{~mL}$ bottles for analysis.

\section{Classical parameters}

The wines were analysed according to the methods of the International Organisation of Vine and Wine (OIV). A wine sample of $125 \mathrm{~mL}$ was used to determine the alcohol content, titratable acidity and $\mathrm{pH}$. For each parameter, all analyses were performed in triplicate 30 days after blending.

\section{Extraction and chromatographic analysis of wine volatiles}

Wine volatile compounds were analysed by GC-MS according to the method proposed by Oliveira et al. (2006).

To a $10 \mathrm{~mL}$ culture tube, $8 \mathrm{~mL}$ of wine, $2.4 \mu \mathrm{g}$ of internal standard 4-nonanol (Merck 818 773, Darmstadt, Germany) and a magnetic stir bar $(22.2 \mathrm{~mm} \times 4.8 \mathrm{~mm})$ were added. Extraction was performed by stirring the sample with 400 $\mu \mathrm{L}$ of dichloromethane (Merck 1.06054) for $15 \mathrm{~min}$. After cooling at $0^{\circ} \mathrm{C}$ for $10 \mathrm{~min}$, the magnetic stir bar was removed and the organic phase was obtained by centrifugation $(2$

TABLE 1

Composition of single and blend wines (in \%)

\begin{tabular}{|c|c|c|c|c|c|}
\hline Wines & Code & Albariño & Loureira & Caiño Blanco & Godello \\
\hline 1 & $\mathrm{AL}$ & 100 & - & - & - \\
\hline 2 & LO & - & 100 & - & - \\
\hline 3 & AL-CB & 83 & - & 17 & - \\
\hline 4 & AL-LO-CB & 48 & 32 & 20 & - \\
\hline 5 & LO-CB & - & 50 & 50 & - \\
\hline 6 & LO-CB-GO & - & 35 & 35 & 30 \\
\hline
\end{tabular}

AL: Albariño; LO: Loureira; AL-CB: Albariño+Caiño Blanco; AL-LO-CB: Albariño+Loureira+Caiño Blanco; LO-CB: Loureira+Caiño Blanco; LO-CB-GO: Loureira+Caiño Blanco+Godello

TABLE 2

Results of chemical analysis of single and blended wines

\begin{tabular}{lllll}
\hline Wines & Code & Ethanol (\% vol) & Total acidity (g/L) & pH \\
\hline Albariño & AL & 13.82 & 7.05 & 3.45 \\
Loureiro & LO & 12.75 & 7.20 & 3.25 \\
Albariño-Caiño Blanco & AL-CB & 13.03 & 8.25 & 3.37 \\
Albariño-Loureiro-Caiño Blanco & AL-LO-CB & 12.53 & 7.35 & 3.33 \\
Loureiro-Caiño Blanco & LO-CB & 13.10 & 7.80 & 3.32 \\
Loureira-Caiño Blanco-Godello & LO-CB-GO & 13.58 & 7.05 & 3.26 \\
\hline
\end{tabular}




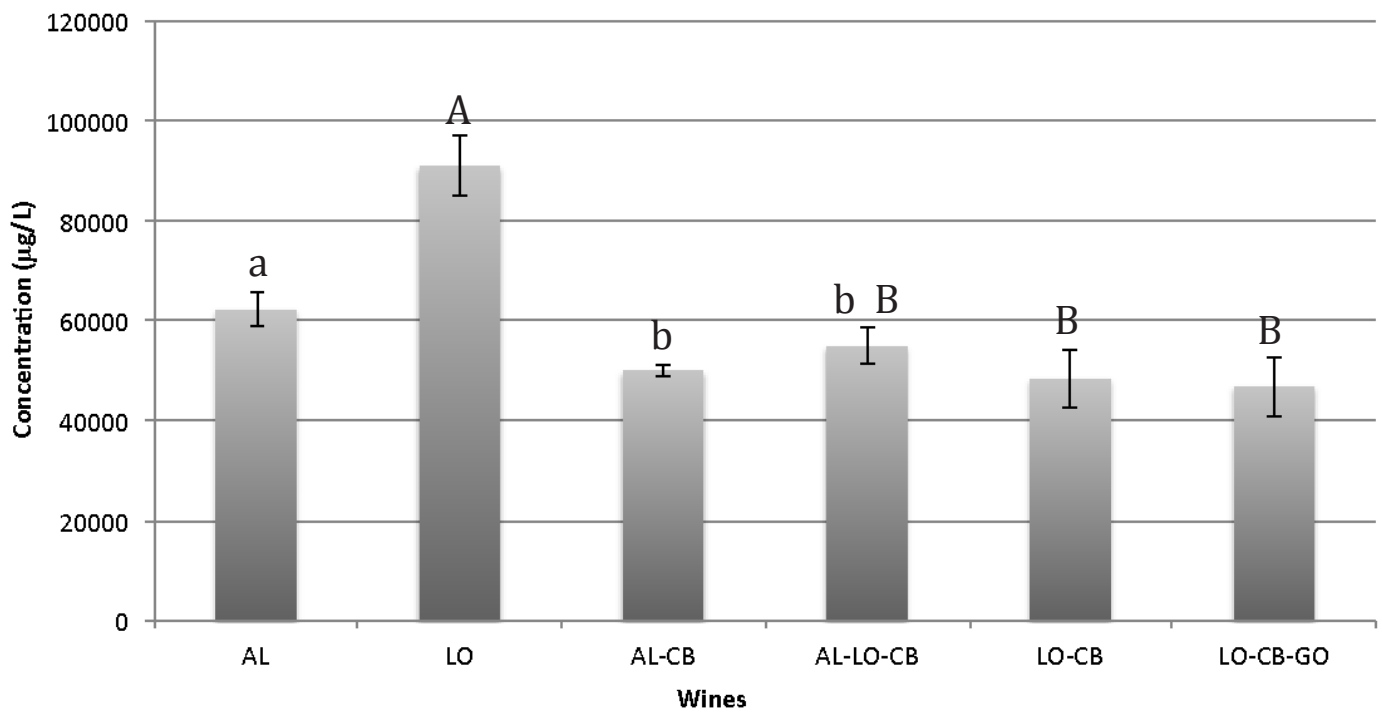

FIGURE 1

Total volatile composition of monovarietal and blended wines

AL: Albariño; LO: Loureira; AL-CB: Albariño+Caiño Blanco; AL-LO-CB: Albariño+Loureira+Caiño Blanco; LO-CB: Loureira+Caiño Blanco; LO-CB-GO: Loureira+Caiño Blanco+Godello. Different uppercase or lowercase letters show significant differences among wines by Fisher's least significant difference (LSD) at $P<0.05$

$\left.948 \times g, 5 \mathrm{~min}, 4^{\circ} \mathrm{C}\right)$, with the extract being recovered in a vial using a Pasteur pipette. The aromatic extract was then dried with anhydrous sodium sulphate (Merck 1.06649) and collected again in a new vial.

Separation was performed using a gas chromatograph (Agilent GC 6890 N, CA, USA) equipped with a mass spectrometer detector (Agilent MS 5975C, CA, USA).

A $3 \mu \mathrm{L}$ injection was made into a capillary column coated with ZP-Wax $52 \mathrm{CB}(50 \mathrm{~m} \times 0.25 \mathrm{~mm}$ i.d., $0.25 \mu \mathrm{m}$ film thickness, Phenomenex). The injector temperature was $250^{\circ} \mathrm{C}$ and the detector temperature was $280^{\circ} \mathrm{C}$. The oven temperature was maintained at $50^{\circ} \mathrm{C}$ for $5 \mathrm{~min}$, and then programmed to rise from 50 to $220^{\circ} \mathrm{C}$ at $3{ }^{\circ} \mathrm{C} / \mathrm{min}$, and finally from 220 to $250^{\circ} \mathrm{C}$ at $5^{\circ} \mathrm{C} / \mathrm{min}$. The carrier gas was helium 55 (Air Liquide, Maia, Portugal) at $103 \mathrm{kPa}$, and the split vent was set to $13 \mathrm{~mL} / \mathrm{min}$. Each $3 \mu \mathrm{L}$ extract was injected in splitless mode (for $15 \mathrm{~s}$ ). Identification was performed using Wsearch 321.6 free software (http://www.wsearch.com.au) by comparing the mass spectra and retention indices with those of pure standard compounds. All of the compounds were quantified as 4-nonanol equivalents.

\section{Odour activity value}

The OAV, which is an indicator of the importance of a specific compound to the odour of a sample, was determined to evaluate the contribution of each chemical compound to the sensory perception of the wine. It was calculated as the ratio between the concentration of an individual compound and the perception threshold found in the literature (Etievant, 1991; Guth, 1997; Ferreira et al., 2000; Lopez et al., 2002; Escudero et al., 2007; Ferreira et al., 2009).

\section{Statistical analysis}

Data were analysed using XLstat-Pro (2007 Version,
Addinsoft, Paris, France). Significant differences among wines for each of the parameters analysed were assessed using one-way analysis (ANOVA). Fisher's least significant difference (LSD) means comparison test $(p<0.05)$ was performed. Discriminant analyses (DA) were performed to establish the relationship between the parameter analysed and the type of single or blended wine.

\section{RESULTS AND DISCUSSION}

\section{Total volatile composition of the wines}

The chemical parameters of the wines are shown in Table 2. The $\mathrm{pH}$ values of the wines were between 3.26 and 3.45, and the titratable acidity values were between 7.05 and $8.25 \mathrm{~g} / \mathrm{L}$. The alcohol contents were between $12.53 \%(\mathrm{v} / \mathrm{v})$ for ALLO-CB and $13.10 \%(\mathrm{v} / \mathrm{v})$ for LO-CB. Albariño and Loureira single wines showed alcohol contents of $13.82 \%(\mathrm{v} / \mathrm{v})$ and $12.75 \%(\mathrm{v} / \mathrm{v})$ respectively.

Fig. 1 shows the mean and standard deviation of the total volatile composition of the single (Albariño and Loureira) and blended wines (83:17 AL-CB, 48:32:20 ALLO-CB, 50:50 LO-CB and 35:35:30 LO-CB-GO). Fisher's LSD of the mean scores for the total volatile composition was calculated to show differences among the single and blended wines. The monovarietal Loureira and Albariño wines had higher total volatile concentrations compared to the blended wines (Fig. 1), with the monovarietal Loureira wine exhibiting the highest value $(91.036 \mu \mathrm{g} / \mathrm{L})$, followed by the Albariño single wine $(62.272 \mu \mathrm{g} / \mathrm{L})$. The total volatile concentration of the single wines changed when they were blended. Compared with Albariño, the blended wines (93:17 AL-CB and 48:32:20 AL-LO-CB) showed significant decreases $(p<0.05)$ in total volatile concentrations. In the same way, significant decreases $(p<0.05)$ were noted for the blended wines (48:32:20 AL-LO-CB, 50:50 LO-CB 
and 35:35:30 LO-CB-GO) compared with Loureira. This decrease in total volatile concentrations for the blended wines $v s$. the single wines was independent of the proportion of each wine cultivar and could be attributed to a dilution effect.

Table 3 presents the volatile concentrations (expressed as $\mu \mathrm{g} / \mathrm{L}$ ) quantified in the single and blended wines arranged into six chemical families (alcohols, $\mathrm{C}_{6}$-compounds, ethyl esters, terpenes-norisoprenoids, volatile acids and phenol volatiles). Fisher's LSD of the mean scores for all families of volatile compounds was calculated to compare the volatile composition of the single $v s$. the blended wines (Table 3 ). The highest concentration of all families of volatile compounds was exhibited for Loureira wines, except for phenol volatiles and volatile fatty acids, for which the blended wines 50:50 LO-CB and 48:32:20 AL-LO-CB showed the highest concentrations respectively. The superiority of the total volatile composition of Loureira wines vs. other Galician white wines, such as Albariño, Treixadura, Blanco lexítimo and Torrontés, has been demonstrated previously (Vilanova et al., 2013). The study by Vilanova et al. (2013) also showed no significant differences in volatile acids between Albariño and Loureira wines; however, Albariño wine had a higher phenol volatile content than the Loureira wine. Oliveira et al. (2008) showed that wines from the two cultivars, Albariño and Loureira, had a similar composition of volatiles. However, Loureira wine was richer in varietal compounds compared to Albariño wine with respect to $\mathrm{C}_{6}$-compounds and monoterpenic compounds, in contrast to the volatile phenols (Oliveira et al., 2008).

When the comparison was performed among Albariño single wine $v s$. blended wines (83:17 AL-CB and 48:32:20 AL-LO-CA), Fisher's LSD analysis showed significant differences $(p<0.05)$ for all volatile families, with the exception of the ethyl esters (Table 3 ). Albariño wine only showed the highest values for alcohols; however, the blended wine 83:17 AL-CB showed the highest concentration of $\mathrm{C}_{6}$ compounds and phenol volatiles. Blend 48:32:20 AL-LO$\mathrm{CB}$ had the highest concentrations of volatile fatty acids and terpenes $+\mathrm{C}_{13}$-norisoprenoids.

By contrast, when the comparison was carried out among single Loureira wine vs. blended wines (48:32:20 AL-LOCB, 83:17 LO-CB and 35:35:30 LO-CB-GO), the results showed the superiority of Loureira single wines vs. blended wines for all families, except for the phenol volatiles, for which 50:50 LO-CB showed the highest content (Table 3). Godello wines were characterised by the lowest values of monoterpenes when compared with Albariño and Loureira wines (Versini et al., 1994).

\section{Major and minor volatile compounds}

Table 4 presents the mean concentrations (expressed as $\mu \mathrm{g} / \mathrm{L}$ ) of 32 free volatile compounds quantified in the single and blended wines. The results of Fisher's LSD to compare the means of individual volatile compounds of Albariño and Loureira single wines vs. blended wines are also included in Table 4.

\section{Alcohols}

This was the most prevalent group of volatile compounds, with a total of nine compounds quantified, among which 4-methyl-2-pentanol and 2- phenylethanol were the most abundant alcohols in all wines (Table 4).

Loureira wines showed the highest concentration of all alcohols, except for 1-butanol. Similar results were found for Loureira wines by Vilanova et al. (2013). When the comparison was performed between Albariño single wine vs. the blended wines (83:17 AL-CB and 48:32:20 AL-LO-CB), Fisher's LSD analysis identified significant differences $(p<0.05)$ for all alcohols, except 4-methyl-2pentanol and methionol. All alcohols quantified showed their highest concentration in Albariño single wine vs. blended wines, except for 2-phenylethanol, which was highest in the blended wine 48:32:20 AL-LO-CB. The high concentration of 2-phenylethanol in the Loureira single wine could explain the high level of this compound in the 48:32:20 AL-LO-CB blended wine.

When the comparison was carried out among single Loureira wine vs. blended wines (48:32:20 AL-LO-CB, 50:50 LO-CB and 35:35:30 LO-CB-GO), the results showed the superiority of Loureira single wines vs. blended wines for all alcohols, with significant differences among the wines.

TABLE 3

Volatile composition by families of single and blended wines

\begin{tabular}{|c|c|c|c|c|c|c|c|c|}
\hline Families & $\mathrm{AL}$ & $\mathrm{LO}$ & AL-CB & $\begin{array}{l}\text { AL-LO- } \\
\text { CB }\end{array}$ & $\mathrm{LO}-\mathrm{CB}$ & $\begin{array}{l}\text { LO-CB- } \\
\text { GO }\end{array}$ & $\begin{array}{l}\mathrm{AL} / \mathrm{AL}- \\
\mathrm{CB} / \mathrm{AL}- \\
\mathrm{LO}-\mathrm{CB}\end{array}$ & $\begin{array}{l}\text { LO/AL- } \\
\mathrm{LO}-\mathrm{CB} / \\
\mathrm{LO}-\mathrm{CB} / \\
\mathrm{LO}-\mathrm{CB}-\mathrm{GO}\end{array}$ \\
\hline Alcohols & 38550.50 & 64114.77 & 28492.43 & 32129.16 & 31059.03 & 25749.14 & $a-c-b$ & $a-b-b-b$ \\
\hline $\mathrm{C}_{6}$-alcohols & 586.85 & 1284.76 & 730.96 & 600.48 & 664.67 & 747.70 & $b-a-b$ & $a-b-b-b$ \\
\hline Ethyl esters & 13133.83 & 14578.23 & 12656.47 & 9697.79 & 10067.07 & 9955.76 & ns & $a-b-b-b$ \\
\hline Volatile acids & 9806.55 & 10855.50 & 7854.25 & 12273.29 & 6166.14 & 10212.40 & $b-b-a$ & $a-a-b-a$ \\
\hline $\begin{array}{l}\text { Terpenes + C13- } \\
\text { norisoprenoids }\end{array}$ & 65.65 & 156.44 & 45.78 & 117.07 & 104.40 & 49.74 & $b-b-a$ & $a-a b-a b-b$ \\
\hline Phenol volatiles & 129.03 & 46.41 & 276.98 & 191.69 & 249.03 & 126.49 & $b-a-b$ & $d-b-a-c$ \\
\hline
\end{tabular}

AL: Albariño; LO: Loureira; AL-CB: Albariño+Caiño Blanco; AL-LO-CB: Albariño+Loureira+Caiño Blanco; LO-CB: Loureira+Caiño Blanco; LO-CB-GO: Loureira+Caiño Blanco+Godello. Different letters in each row indicate a significant difference among wines by Fisher's least significant difference (LSD) at $P<0.05$; ns: not significant 


\section{Ethyl esters and acetates}

This group was represented by 10 compounds, which showed high contents in all wines analysed, with 3-methylbutyl acetate having the highest concentration in all wines (Table 4). The comparison between Albariño wines vs. blended wines (83:17 AL-CB and 48:32:20 AL-LO-CB) showed significant differences for eight compounds. In this case, Albariño single wine had the highest concentrations of ethyl esters and acetates, except for ethyl octanoate and ethyl decanoate. These compounds exhibited the highest values in the blended wine 35:35:30 LO-CB-GO. The influence of Caiño Blanco produced an increase in 2-phenyl ethyl acetate in the blended wines 83:17 AL-CB and 35:35:30 AL-LO-CB $v s$. Albariño monovarietal wine. Loureira wine showed the highest concentration of ethyl esters compared with those in the blended wines (48:32:20 AL-LO-CB, 50:50 LO-CB and 35:35:30 LO-CB-GO). However, no significant differences were observed between Loureira and blended wines for ethyl 2-methyl butanoate, ethyl lactate, diethyl succinate and 3-methylbutyl acetate. Vilanova et al. (2013) showed that Loureira wines had a higher 2-ethyl methylbutanoate content compared to other white wines from Galicia, such as Albariño, Treixadura, Blanco lexítimo and Torrontés.

\section{$C_{6}$-compounds}

This group was represented by three compounds (Table 4). Loureira monovarietal wines had the highest concentration of all three compounds; however, the blended wine with Loureira (48:32:20 AL-LO-CB) showed only an increase in E-3-hexen-ol vs. the Albariño wine. When the comparison was made between Loureira single wine and blended wines (48:32:20 AL-LO-CB, 83:17 LO-CB and 35:35:30 LO-CB$\mathrm{GO})$, the decreases in the contents of $\mathrm{C}_{6}$-compounds in the blended wines indicated a dilution effect. Rapp et al. (1993) showed that the contents of $E$-3-hexenol and its isomer Z-3-hexenol are the most important analytical parameters to discriminate monovarietal wines of Riesling, MüllerThurgau, Kerner, Scheurebe, Ehrenfelser and Bacchus. Oliveira et al. (2006) concluded that the $E$-3-hexenol/Z3-hexenol ratio could discriminate Loureiro clearly from Alvarinho wines from Northern Portugal.

\section{Volatile fatty acids}

Seven compounds in this group were identified and quantified (Table 4). Hexanoic, octanoic and decanoic acids showed the highest values among all wines analysed. The comparison among single Albariño vs. blended wines (83:17 AL-CB and 48:32:20 AL-LO-CB) showed that 2+3-methyl butyric acid, octanoic acid and decanoic acid levels were the highest in monovarietal Albariño and 48:32:20 AL-LO-CB blended wines. In the comparison between Loureira single wine and the blended wines (48:32:20 AL-LO-CB, 50:50 LO-CB and 35:35:30 LO-CB-GO), the highest concentration of the volatile fatty acids family was observed in Loureira single wine. The blended wine 50:50 LO-CB showed the lowest concentration of all volatile fatty acids. In a previous study, only 3-methylbutyric and decanoic acids showed significant differences among the white wines from Galicia, and were present at higher concentrations in Loureira, Treixadura and Blanco lexítimo wines vs. Albariño and Torrontés white wines (Vilanova et al., 2013).

Among terpenes and $\mathrm{C}_{13}$-norisoprenoids, only linalool showed significant differences among the wines, with Loureira single wine showing the highest concentration (Table 4). Therefore, the blended wine with Loureira (48:32:20 AL-LO-CB) had an increased linalool content compared with the Albariño monovarietal wine. Vilanova et al. (2013) showed that Loureira and Albariño wines had similar volatile compositions. However, regarding varietal compounds, Loureira wines were richer in monoterpene compounds compared with Albariño wines (Genisheva \& Oliveira 2009; Vilanova et al., 2013). Other studies have indicated that Loureira can be classified among the monoterpene-dependent aromatic varieties, and that Loureira and Albariño varieties have an important reserve of volatile compounds that can be exploited technologically (Oliveira et al., 2000). Monoterpenes are the source of floral and fruity characteristics of wines made from grape varieties such Muscat and Gewürztraminer, and to a lesser extent those made from Riesling, Albariño and Loureiro (Wilson et al., 1986; Versini et al., 1994; Muñoz-Organero et al., 1998; Bureau et al., 2000; Oliveira et al., 2008).

Oliveira et al. (2008) concluded that terpenols seem to be more important in Loureiro wines and the $\mathrm{C}_{13}$-norisoprenoids are more important in Albariño wines. Because of their high concentrations and low aroma thresholds, terpenes and $\mathrm{C}_{13}$ norisoprenoids are the principal components responsible for the characteristic aroma of a wine (Carballeira et al., 2001).

Phenol volatiles were represented by only one compound (4-vinylguaiacol), for which the blended wines 83:17 AL-CB and 50:50 LO-CB exhibited higher concentrations than the Albariño and Loureira single wines respectively (Table 4). Therefore, blending Caiño Blanco (CB) with Loureira (LO) and Albariño (AL) induced an increase in 4-vinylguaiacol. In other studies, no significant differences were shown between Albariño and Loureira wines for 4-vinylguaiacol (Vilanova et al., 2013).

\section{Odour activity value (OAV)}

To assess the influence of each single volatile compound on the sensory perception of the wines, the OAV was calculated as the ratio between the concentration of the compound and its odour threshold. The results in Table 5 show that, among the 32 compounds analysed, only 17 reached a concentration above the odour threshold $(\mathrm{OAV}>1)$ in at least one wine. Thirteen volatile compounds were found above the threshold in all samples. Loureira single wine and 50:50 LO-CB blended wine exhibited the highest total OAVs. For all wines, the highest OAV was exhibited by $\beta$-damascenone (apple aroma), which was highest in the blend LO-CB (OAV $=1$ 710.48). Ethyl hexanoate (green apple), ethyl octanoate (apple) and 3-methyl butyl acetate (banana) also exhibited high OAVs for all wines, where the highest values were exhibited by Albariño single wine (3-methylbutyl acetate) and Loureira single wine (ethyl hexanoate and octanoate). Loureira exhibited the highest OAV for linalool (3.84). Linalool contributes to the fruity and floral aroma of wines, with an odour threshold of $25 \mathrm{mg} / \mathrm{L}$ (Ribéreau-Gayon et al., 2000; Escudero et al., 2004).

Falqué et al. (2001) showed the OAVs of Galician white 
TABLE 4

Mean values of volatile compounds $(\mu \mathrm{g} / \mathrm{L})$ of Albariño, Loureira and blended wines.

\begin{tabular}{|c|c|c|c|c|c|c|c|c|}
\hline Volatile compounds & $\mathbf{A L}$ & LO & AL-CB & $\begin{array}{l}\text { AL-LO- } \\
\text { CB }\end{array}$ & LO-CB & $\begin{array}{l}\text { LO-CB- } \\
\text { GO }\end{array}$ & $\begin{array}{l}\text { AL/AL- } \\
\text { CB /AL- } \\
\text { LO-CB }\end{array}$ & $\begin{array}{l}\text { LO/AL- } \\
\text { LO-CB/ } \\
\text { LO-CB/ } \\
\text { LO-CB- } \\
\text { GO }\end{array}$ \\
\hline 2-Methyl-1-propanol & 1130.49 & 1886.71 & 371.67 & 734.53 & 783.03 & 595.82 & $\mathrm{a} / \mathrm{b} / \mathrm{ab}$ & $a-b-b-b-b$ \\
\hline 1-Butanol & 47.41 & 36.98 & 21.17 & 22.38 & 16.37 & 25.80 & $a-b-b$ & ns \\
\hline 4-Methyl-2-pentanol & 62.20 & 108.17 & 64.92 & 45.26 & 58.74 & 67.55 & ns & $a-b-b-b$ \\
\hline 2+3-Methyl-1-butanol & 31195.55 & 45373.39 & 20892.83 & 22854.42 & 14193.10 & 18542.83 & $a-b-b$ & $a-b-b-b$ \\
\hline 2-Methyl-1-pentanol & 105.69 & 106.17 & 99.21 & 90.11 & 87.97 & 99.70 & $a-a b-b$ & ns \\
\hline 3-Methyl-1-pentanol & 29.72 & 51.31 & 20.88 & 21.38 & 40.81 & 25.64 & $a-b-b$ & $a-b-a-b$ \\
\hline 2-Octanol & 235.53 & 240.00 & 225.30 & 214.33 & 206.68 & 230.22 & $a-b-c$ & $a-b-b-a$ \\
\hline Methionol & 21.16 & 38.70 & 25.92 & 27.00 & 42.74 & 24.47 & ns & $a-b-a-b$ \\
\hline 2-Phenylethanol & 5722.76 & 16273.34 & 6770.53 & 8119.75 & 15629.59 & 6137.12 & $c-b-a$ & $a-b-a-b$ \\
\hline 1-Hexanol & 545.65 & 1154.85 & 678.10 & 554.64 & 614.45 & 687.38 & $b-a-b$ & $a-b-b-b$ \\
\hline E-3-Hexen-1-ol & 17.60 & 105.08 & 42.46 & 40.52 & 35.94 & 47.45 & $b-a-a$ & $a-b-b-b$ \\
\hline Z-3-Hexen-1-ol & 23.60 & 24.83 & 10.40 & 10.65 & 21.42 & 12.88 & $a-b-b$ & ns \\
\hline Ethyl butyrate & 394.53 & 379.92 & 292.35 & 220.05 & 279.75 & 288.13 & $a-a b-b$ & $a-b-a b-a b$ \\
\hline Ethyl 2-methylbutanoate & 320.24 & 369.17 & 198.82 & 223.99 & 206.71 & 149.94 & ns & ns \\
\hline Ethyl hexanoate & 1291.94 & 1852.34 & 1001.49 & 1123.05 & 1052.96 & 999.22 & $a-c-b$ & $a-b-b-b$ \\
\hline Ethyl lactate & 98.44 & 80.42 & 59.85 & 68.91 & 68.13 & 77.92 & $a-b-b$ & ns \\
\hline Ethyl octanoate & 1144.43 & 2375.71 & 1684.60 & 2078.47 & 1907.53 & 1148.49 & $c-b-a$ & $a-b-b-c$ \\
\hline Ethyl decanoate & 410.15 & 599.80 & 420.13 & 735.77 & 717.76 & 337.37 & $b-b-a$ & $a b-a-a-b$ \\
\hline Diethyl succinate & 25.38 & nd & 16.95 & 9.56 & nd & 19.53 & ns & ns \\
\hline 3-Methyl butyl acetate & 8501.41 & 7341.07 & 7676.83 & 4138.94 & 4549.29 & 5946.35 & $a-a b-b$ & ns \\
\hline Hexyl acetate & 485.49 & 741.83 & 637.37 & 407.19 & 386.56 & 537.71 & $b-a-c$ & $a-b c-c-b$ \\
\hline 2-Phenyl ethyl acetate & 478.73 & 837.97 & 679.39 & 691.85 & 898.36 & 451.09 & b-a-a & $a-a-a-b$ \\
\hline Butyric acid & 43.01 & 27.04 & 29.41 & 12.59 & 22.62 & 38.52 & $a-a b-b$ & $a b-b-b-a$ \\
\hline 2+3-Methylbutyric acid & 98.66 & 130.08 & 66.01 & 83.39 & 119.98 & 104.23 & $a-b-a b$ & $a-c-a b-b c$ \\
\hline Hexanoic acid & 1488.70 & 1990.93 & 1557.99 & 1849.75 & 1317.77 & 1950.84 & ns & $a-a b-b-a$ \\
\hline Heptanoic acid & 321.83 & 330.73 & 351.93 & 369.45 & 214.88 & 370.27 & ns & $a-a-b-a$ \\
\hline Octanoic acid & 5716.23 & 6354.93 & 4543.72 & 6994.04 & 3577.47 & 5842.67 & $a b-b-a$ & $a-a-b-a$ \\
\hline Decanoic acid & 1816.31 & 1851.88 & 1096.15 & 2556.10 & 821.85 & 1712.41 & $a b-b-a$ & $a b-a-b-a b$ \\
\hline Dodecanoic acid & 321.80 & 169.91 & 209.03 & 407.98 & 99.11 & 193.47 & ns & ns \\
\hline Linalool & 9.97 & 96.03 & 9.77 & 48.00 & 18.88 & 3.79 & $b-b-a$ & $a-b-b c-c$ \\
\hline$\beta$-Damascenone & 55.68 & 60.41 & 36.01 & 69.08 & 85.52 & 45.95 & ns & ns \\
\hline 4-Vinylguaiacol & 129.03 & 46.41 & 276.98 & 191.69 & 249.03 & 126.49 & $b-a-b$ & $d-b-a-c$ \\
\hline
\end{tabular}

AL: Albariño; LO: Loureira; AL-CB: Albariño+Caiño Blanco; AL-LO-CB: Albariño+Loureira+Caiño Blanco; LO-CB: Loureira+Caiño Blanco; LO-CB-GO: Loureira+Caiño Blanco+Godello. Different letters in each row indicate a significant difference among wines by Fisher's least significant difference (LSD) at $P<0.05$; ns: not significant; nd: not determined

grape varieties, where they concluded that a high level of OAV of Linalool in Loureira could explain the floral and flavour descriptors of this cultivar. Other study showed linalool as the only terpene with $\mathrm{OAV}>1$, where Loureira showed the highest values (Vilanova et al., 2013). In agreement with Vilanova et al. (2013), 2-phenylethanol also showed the highest OAV for Loureira.

Discriminant analysis (DA)

Two DAs were used to discriminate the single wines from the blended wines on the basis of their volatile composition (Fig. 2).

The first DA (Fig. 2a) shows the discriminant analyses of Albariño single wine and the blended wines in terms of volatile compound families, where the wines were clearly separated by two canonical discriminating functions. The first function separated single-wine Albariño from the blended wines (Albariño-Loureira-Caiño Blanco and Albariño-Caiño Blanco), explaining $99.78 \%$ of the variance. The second function explained $100 \%$ of the accumulated variance. 
a)
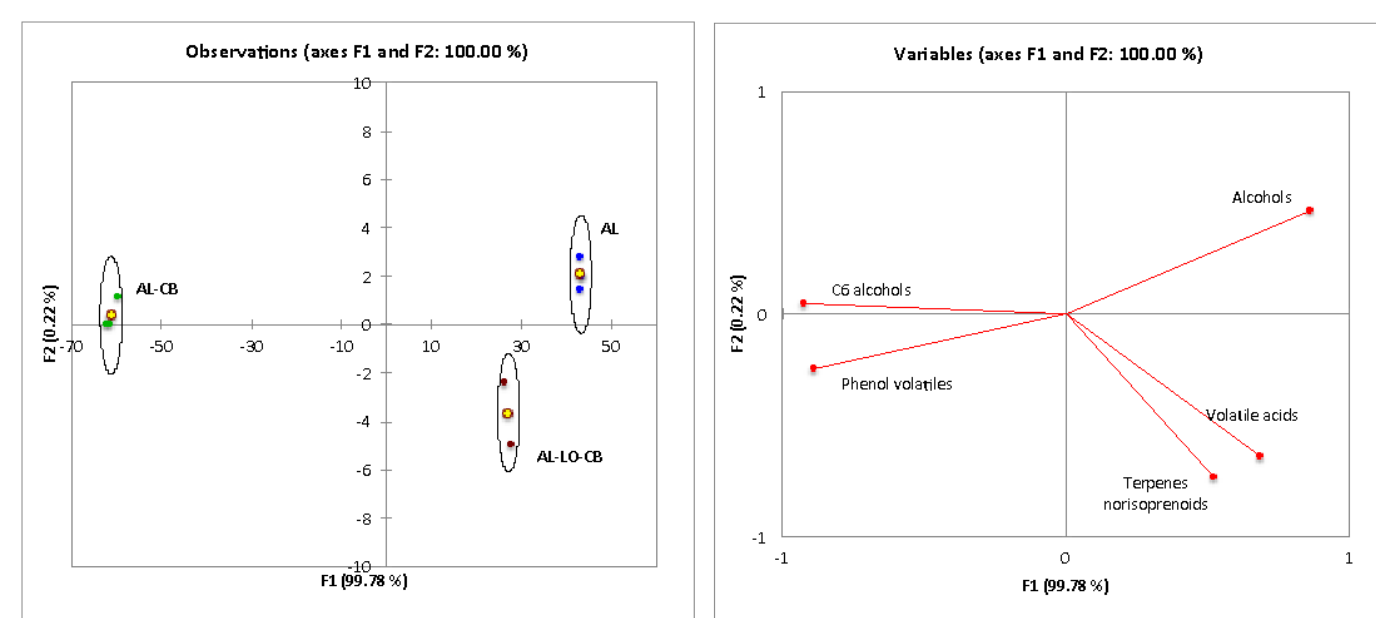

b)
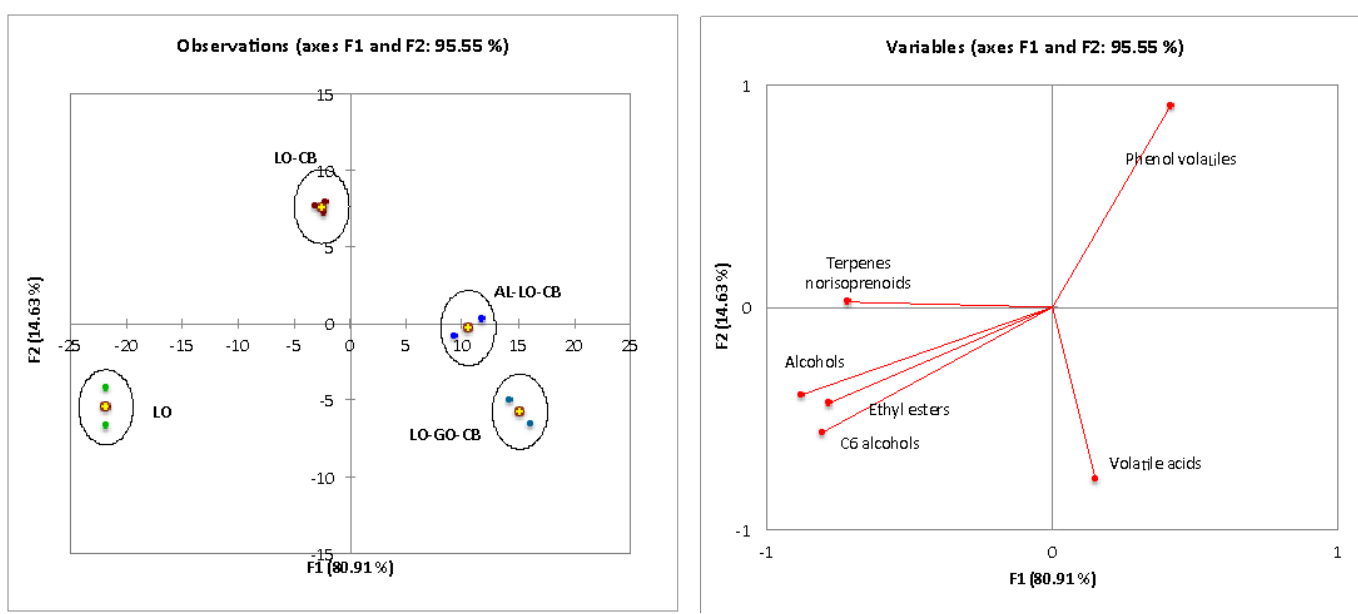

FIGURE 2

Discriminant analysis (DA) of single Albariño (a) and Loureira (b) wines vs. blended wines for volatile composition by families. AL: Albariño; LO: Loureira; AL-CB: Albariño+Caiño Blanco; AL-LO-CB: Albariño+Loureira+Caiño Blanco; LO-CB: Loureira+Caiño Blanco; LO-CB-GO: Loureira+Caiño Blanco+Godello

The second DA (Fig. 2b) shows the discriminant analysis of Loureira single wine and the blended wines in terms of the concentrations of volatile compounds, where the Loureira single wine and blended wines (Loureira-Caiño Blanco, Albariño-Loureira-Caiño Blanco and LoureiraCaiño Blanco-Godello) were clearly separated by the two canonical discriminating functions. The first function explained $80.91 \%$ and the second explained $100 \%$ of the accumulated variance.

In both discriminant analyses, a good separation was observed between the single and blended wines. When DA was carried out for single-wine Albariño and the blends, $\mathrm{C}_{6}$-alcohols and phenol volatiles were the variables that contributed most to this differentiation, whereas terpenes and $\mathrm{C}_{13}$-norisoprenoids were the variables that contributed to the differentiation between Loureira single wine and the blended wines.

The proportion of the wine used in the blended wines also made an important contribution to differentiation of the wines. The incorporation of Loureira wine (32\%) to the blend with Albariño wine (AL-LO-CB) led to a higher concentration of terpenes and $\mathrm{C}_{13}$-norisoprenoids because of the importance of those families to the Loureira volatile composition, mainly represented by linalool (Fig. 2a). In contrast, $50 \%$ of Loureira wine in the blend with $50 \%$ of Caiño Blanco wine (LO-CB) resulted in higher concentrations of terpenes and $\mathrm{C}_{13}$-norisoprenoids compared with the other blended wines (48:32:20 AL-LO-CB and 35:35:30 AL-LOGO), with Loureira contributing a minor proportion $(32 \%$ and $35 \%$ respectively).

\section{CONCLUSIONS}

This study has demonstrated the effects of blending on the volatiles of Albariño and Loureira wines. Wines obtained from blending led to changes in volatile composition, increasing the content of some volatile compounds. However, monovarietal Loureira and Albariño wines showed the highest total volatile concentrations. Monovarietal Loureira wines exhibited the highest content of volatile compounds, characterised by higher levels of terpenoids, especially linalool. Therefore, wine obtained from blending with Loureira showed increases in the concentrations of terpenes $+\mathrm{C}_{13}$-norisoprenoids. The OAV analysis indicated the aromatic superiority of Loureira single wines and 50:50 Loureira-Caiño Blanco blended wines vs. Albariño and the other blended wines. Blended wines with a high proportion 


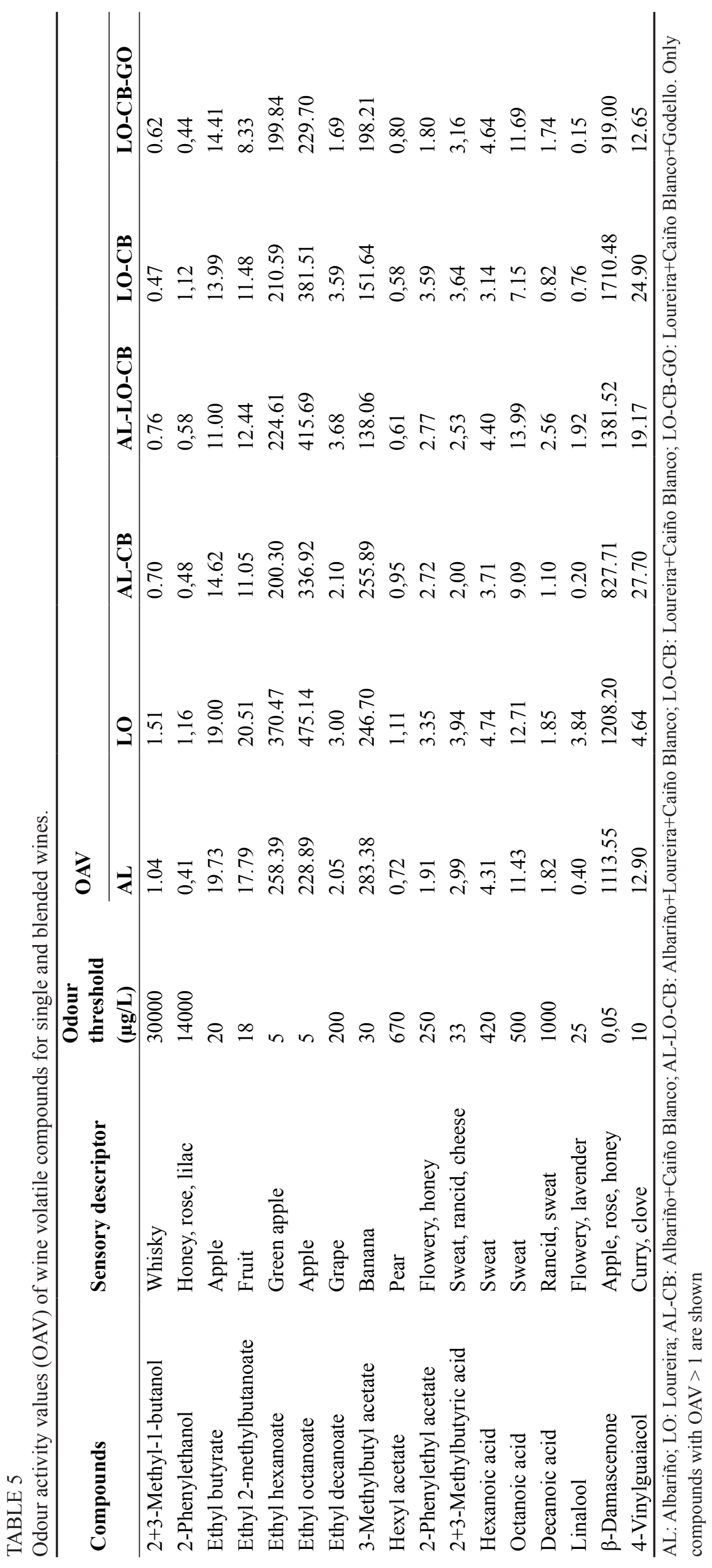


of Loureira are likely to have more complex aromas. Loureira blended with Caiño Blanco or in combination with other wines proved to be suitable for blending, as judged by the increase in the varietal composition of the wines.

\section{LITERATURE CITED}

Bureau, S.M., Razungles, A.J. \& Baumes, R.L., 2000. The aroma of Muscat of Frontignan grapes: Effect of the light environment of vine or bunch on volatiles and glycoconjugates. J. Sci. Food Agr. 80(14), 2012-2020

Carballeira, L., Cortés, S., Gil, M.L. \& Fernández, E., 2001. SPE-GC determination of aromatic compounds in two varieties of white grape during ripening. Chromatographia 53(Suppl), 350-355.

Escudero, A., Campo, E., Fariña, L., Cacho, J. \& Ferreira, V., 2007. Analytical characterization of the aroma of five premium red wines. Insights into the role of odor families as the concept of fruitiness of wines. J. Agric. Food Chem. 55, 4501-4510.

Escudero, A., Gogorza, B., Melus, M.A., Ortin, N., Cacho, J. \& Ferreira, V., 2004. Characterization of the aroma of a wine from Macabeo. Key role played by compounds with low odour activity values. J. Agric. Food Chem. $52,3516-3524$

Etievant, P.X., 1991. Wine. In: H. Maarse (ed). Volatile compounds of food and beverages. Marcel Dekker, New York, USA. pp. $483-546$.

Falqué, E., Fernández, E. \& Dubourdieu, D., 2001. Differentiation of white wines by their aromatic index. Talanta 54, 271-281.

Ferreira, V., Lopez, R. \& Cacho, J.F., 2000. Quantitative determination of the odorants of young red wines from different grape varieties. J. Sci. Food Agr. 80, 1659-1667.

Ferreira, V., San Juan, F., Escudero, A., Culleré, L., Fernández-Zurbano, P., Sáenz-Navajas, M.P. et al., 2009. Modeling quality of premium Spanish red wines from gas chromatography- olfactometry data. J. Agric. Food Chem. 57(16), 7490-7498.

Francis, I.L. \& Newton, J.L., 2005. Determining wine aroma from compositional data. Aust. J. Grape Wine Res. 11, 114-126.

Genisheva, Z. \& Oliveira, J.M., 2009. Monoterpenic characterization of white cultivars from Vinhos Verdes Appellation of Origin (North Portugal). J. Inst. Brew. 115, 308-317.

Guth, H., 1997. Quantification and sensory studies of character impact odorants of different white wine varieties. J. Agric. Food Chem. 45, 30273032

Herderich, M., Barter, S., Black, C.A., Bramley, R., Capone, D., Dry, P., Siebert, T. \& Zhang, P., 2015. Terroir effects on grape and wine aroma compounds. In: Advances in Wine Research. ACS Symposium Series, vol. 1203. American Chemical Society. pp. $131-146$.

Hopfer, H., Ebeler, S.E. \& Heymann H., 2012. How blending affects the sensory and chemical properties of red wine. Am. J. Enol. Vitic. 63(3), 313324 .

Lopez, R., Aznar, M., Cacho, J. \& Ferreira, V., 2002. Quantitative determination of minor and trace volatile compounds in wine by solid-phase extraction and gas chromatography with mass spectrometric detection. J. Chromatogr. A, 966, 166-177.
Losada, M., Andrés, J., Cacho, J., Revilla, E. \& López, J.F., 2011. Influence of some prefermentative treatments on aroma composition and sensory evaluation of White Godello wines. Food Chem. 125, 884-891.

Muñoz-Organero, G., Ortiz, J.M., Cabellos, J.M. \& Arroyo, T., 1998. Algunos compuestos aromáticos en el mosto de moscatel y de otras variedades de vid. Viticultura y Enología Profesional, 56, 48-55.

Oliveira, J.M., Araújo, I.M., Pereira, O.M., Maia, J.S., Amaral, A.J. \& Maia, M.O., 2004. Characterization and differentiation of five Vinhos Verdes grape varieties on the basis of monoterpenic compounds. Anal. Chim. Acta $513,269-275$.

Oliveira, J.M., Faria, M., Sá, F., Barros, F. \& Araujo, I.M., 2006. C6alcohols as varietal markers for assessment of wine origin. Anal. Chim. Acta $563,300-309$.

Oliveira, J.M., Maia, M.O., Baumes, R.L. \& Bayonove, C.L., 2000. Free and bound aromatic components of Loureiro and Alvarinho grape varieties from the Vinhos Verdes region. Vitic. Enol. Sci. 55, 13-20.

Oliveira, J.M., Oliveira, P., Baumes, R.L. \& Maia, M.O., 2008. Volatile and glycosidically bound composition of Loureiro and Alvarinho wines. Food Sci. Technol. Int. 14, 341-353.

Rapp, A., Volkmann, C. \& Niebergall, H., 1993. Untersuchung flüchtiger Inhaltsstoffe des Weinaromas: Beitrag zur Sortencharakterisierung von Riesling und Neuzüchtungen mit Riesling-Abstammung. Vitis 32, 171-178.

Ribéreau-Gayon, P., Glories, Y., Maujean, A. \& Dubourdieu, D. (eds). 2000. Varietal aroma. In: Handbook of enology, vol. 2 - The chemistry of wine. Stabilization and treatments. John Wiley \& Sons Ltd, Hoboken, NJ. pp. 187 $-206$.

Versini, G., Orriols, I. \& Dalla Serra, A., 1994. Aroma components of Galician Albariño, Loureira and Godello wines. Vitis 33, 165-170.

Vilanova, M., 2006. Sensory descriptive analysis and consumer acceptability of Godello wines from Valdeorras Apellation Origen Controlée (northwest Spain). J. Sens. Stud. 21, 262-372.

Vilanova, M. \& Masneuf-Pomarède, I., 2005. Comparison of volatiles in Albariño wine produced by Saccharomyces cerevisiae strain. Ital. J. Food Sci. 17(2), 221-227.

Vilanova, M. \& Vilariño, F., 2006. Influence of geographic origin on aromatic descriptors of Spanish Albariño wines. Flavour Frag. J. 21(2), 373-378.

Vilanova, M., Escudero, A., Graña M. \& Cacho J., 2013. Volatile composition and sensory properties of Vitis vinifera white cultivars from NW Spain. Correlation between sensory and instrumental analysis. Food Res. Int. 54, 562-568.

Vilanova, M., Genisheva, Z., Masa, A. \& Oliveira, J.M., 2010. Correlation between volatile composition and sensory properties in Spanish Albariño wines. Microchem. J. 95(2), 240-246.

Vilanova, M., Zamuz, S., Tardaguila, J. \& Masa, A., 2008. Characterization by descriptive analysis of Vitis vinifera cv. Albariño. J. Sci. Food Agr. 88 , 819-823.

Vilanova, M., Zamuz, S., Vilariño, F. \& Sieiro C., 2007. Effect of terroir on the volatiles of Vitis vinifera cv. Albariño. J. Sci. Food Agr. 87, 1252-1256

Wilson, B., Strauss, C.R. \& Williams, P., 1986. The distribution of free and glycosidically-bound monoterpenes among skin, juice, and pulp fractions of some white grape varieties. Am. J. Enol. Vitic. 37, 107-111. 